\title{
Addressing the Challenges of E-Healthcare in Future Mobile Networks
}

\author{
Safdar Nawaz Khan Marwat ${ }^{1}$, Thomas Pötsch ${ }^{1}$, Yasir Zaki ${ }^{2}$, \\ Thushara Weerawardane ${ }^{3}$, and Carmelita Görg ${ }^{1}$ \\ ${ }^{1}$ ComNets, University of Bremen, Bremen, Germany \\ ${ }^{2}$ New York University Abu Dhabi, Abu Dhabi, United Arab Emirates \\ ${ }^{3}$ Sir John Kotelawala Defense University, Ratmalana, Sri Lanka \\ \{safdar, thp , cg\} @comnets. uni-bremen. de, \\ yz48@nyu.edu, tlw@kdu.ac.lk
}

\begin{abstract}
Machine-to-Machine (M2M) communication is expected to play a major role within the coming years towards the development of e-healthcare applications. The design of cellular networks, such as Long Term Evolution (LTE), is optimized to serve the data traffic of human-based communication with broadband requirements. E-healthcare traffic has different characteristics such as small packet sizes, narrowband requirements and huge number of devices. The focus of this work is to investigate the impact of e-healthcare traffic on LTE cellular networks. We develop a possible future scenario of electrocardiography (ECG) devices performing remote monitoring of patients with mobility support in our LTE simulation model. Regular LTE traffic is also deployed in the network and the influence of the varying ECG traffic is examined. The simulation results indicate that the e-healthcare related data traffic has a drastic influence on regular LTE traffic.
\end{abstract}

Keywords: e-healthcare, M2M, LTE, throughput.

\section{$1 \quad$ Introduction}

Machine-to-Machine (M2M) communication is a global and rapidly developing research area for interconnecting different devices without human intervention. The growth of this area is not only restricted to the diversity and number of future M2M devices, but also the mobile data traffic is expected to grow significantly in future communications [1].

The "Internet of Things" is a new paradigm for interconnecting devices, and the M2M communication is a key area in this field. The authors of [2] anticipate that the "Internet of Things" would extend the existing Internet with a large variety of connected devices. As a result, several application domains would benefit from this concept, for instance supervision in logistical processes, smart metering and monitoring, intelligent transport systems etc.

Recently, researchers have shown an increasing interest in remote monitoring of homes, vehicles and places with M2M devices [3,4,5]. Examples are energy, traffic 
and environmental monitoring. Nowadays, whenever connectivity is debated, the next issue that comes to mind is mobility. Mobile connectivity is almost synonymous with cellular communications. The costs of cellular services experienced dramatic decrease and cellular broadband connectivity became ubiquitous in the recent past. The decrease in the costs and sizes, and the increase of power capabilities of devices with integrated sensors, network interfaces, etc. has paved the way for manufacturers to offer diverse applications and services. Various implementation areas, where those applications can be deployed, benefited from this development.

Long Term Evolution (LTE) and LTE-Advanced (LTE-A) are expected to be the future technologies for providing M2M services. These cellular systems are expected to offer a diverse range of services to M2M applications. M2M applications are generally based on narrowband applications transmitting data frequently or infrequently. The development of LTE was primarily aimed for broadband data services. With narrowband M2M applications, LTE may not achieve spectrum and cost efficiency. Therefore, the integration of M2M communication, with their rather low data rates and small packet sizes but higher number of devices, might have a substantial impact on the LTE system performance.

The concept of e-healthcare is based on establishing a relationship between a healthcare organization and a patient through M2M communication [6]. E-healthcare is defined by the Health Information and Management Systems Society (HIMSS) [7] as "the application of Internet and other related technologies in the healthcare industry to improve the access, efficiency, effectiveness, and quality of clinical and business processes utilized by healthcare organizations, practitioners, patients, and consumers in an effort to improve the health status of patients".

The motivation behind the work of this paper is to study the challenges of providing e-healthcare facilities in future mobile networks. The usage of mobile network resources by mobile e-healthcare devices to monitor the health condition of a patient can have a significant impact on the performance of regular data traffic such as voice, video and file transfer. This paper illustrates the impact of M2M traffic on LTE regular traffic performance by comparing the simulation results of scenarios with varying M2M (e-healthcare) traffic load.

\section{Future Mobile Networks and E-Healthcare}

LTE and LTE-A are the recent standards of wireless communication developed by the Third Generation Partnership Project (3GPP) to fulfill the data volume requirements of cellular mobile users. The objectives of these standards are to increase the peak user throughput, enhance spectral efficiency and reduce latency for broadband services as compared to the previous standards. These recent standards are based on a packet-oriented transmission scheme. The original design goal was to serve cellular devices with data rates of up to $100 \mathrm{Mbps}$ [8]. The access network of LTE, also termed as the Evolved UMTS Terrestrial Radio Access Network (E-UTRAN), consists of only two types of nodes; the eNodeB and the User Equipment (UE). In the radio access network, time and frequency resources are allocated to UEs by the 
Medium Access Control (MAC) layer scheduler of the eNodeB. The scheduler is generally designed to allocate resources for downlink and uplink transmission of regular LTE traffic according to the data buffer sizes of the UEs.

The evolution of mobile networks has led to the notion of providing healthcare and patient monitoring facilities outside the hospital and dispensary premises. Traditionally, the health condition of a patient, suffering from a disease which requires regular check-ups, is monitored on the hospital bed. The patient's temperature, blood pressure, heartbeat rate, etc. are monitored by the staff of the designated hospital ward. Hospital beds and staff are valuable but scarce public resources. The allocation management of these resources has routinely been a challenging task for healthcare organizations, even in advanced countries.

With the recent technological advancements, the topic of patient monitoring from remote locations has been investigated by employing Wireless Local Area Networks (WLANs). The patients can be monitored while at home wearing sensors connected to a WLAN for transferring the health status to the hospital; for example, sending the temperature, electrocardiography (ECG) data, or electronic images of the patient from a remote location using a sensor belt wrapped around the patient's chest. This arrangement provides ease, not only to the healthcare organizations but also, to the patients by significantly decreasing the waiting time in the hospital. Moreover, the domestic routine activities of the patients are not disturbed if they stay at home. The healthcare organizations are expected to take measures for patient treatment only when the sensors detect an unusual health status. This saves healthcare resources. An additional advantage for the patient is to stay away from the hospital environment and reduce the risk of infections and other diseases.

As explained above, mobility is a very special feature of cellular networks and mobility support is a major requirement of several M2M applications. E-healthcare is no exception. It is usually advisable for patients to keep themselves involved in outdoor physical activities like jogging, walking, exercises, etc. In order to perform such activities, the patients are required to leave their home (or office) WLAN coverage areas. In daily life, patients may also be expected to travel by vehicles to go for shopping, picnicking, as well as visiting their relatives and friends; or in critical cases, being taken to hospital by ambulance. In such situations, it is apparent that the home WLAN is not a practicable solution for providing e-healthcare services. In these scenarios, the role of mobile networks becomes vital. The monitoring of patient conditions in mobility scenarios is the focus of this research work.

\section{Literature Review}

Most of the literature available on e-healthcare technologies focuses on indoor scenarios of patient monitoring. The authors in [9] have highlighted the security issues of ehealthcare over mobile networks, and have proposed an architecture to facilitate doctors' and patients' mobility within the hospital. In [10], various M2M implementation scenarios including e-healthcare have been discussed. The authors underline the use of capillary networks and enhanced LTE architecture for M2M communications. In [11], the issues of energy efficiency, reliability and security are elaborated for M2M 
communications and e-healthcare. In [12], the authors suggest a system design for body parameter sensing over GSM/GPRS (Global System for Mobile Communication/General Packet Radio Service) via a modem capable of sending and receiving SMS (Short Message Service). In [13], the authors propose mobile sensor agent and adapter for intelligent M2M communication in e-healthcare. In [14], the integration of vehicular networking and e-healthcare technologies is studied with Bluetooth devices employed at the roadside. The mobile network requirements and issues related to ehealthcare and patient monitoring are discussed in [15]. In [16], two major types of network architectures for e-healthcare are elaborated, i.e. home-oriented architecture and hospital-oriented architecture.

A review of the available literature on this topic reveals that most of the ehealthcare based research is focused on home or hospital scenarios. Only few authors discuss the issues of mobile networks. The discussion on mobile networks is also limited to GSM/GPRS. Our paper highlights the issues of mobile networks under the paradigm of LTE and LTE-A.

\section{$4 \quad$ Remote Health Monitoring}

The health status of a patient can be monitored by providing belts or straps equipped with sensors, to be worn by the patient. The sensors would read the health parameters of the patient regularly and transmit the information over a communication medium to the e-healthcare organization. Based on the received information, the e-healthcare service provider takes remedial actions according to patient conditions. The transmission of health parameters at hospital and home scenarios is possible with the help of capillary networks like WLAN, ZigBee, etc.. However, if the patient is walking down the street or driving a vehicle, the capillary network may not be an appropriate solution for e-healthcare. In such cases, the cellular networks can play a significant role.

The usual health parameters which are frequently required for the evaluation of patient health status are temperature, blood pressure, heartbeat rate, etc. The temperature and blood pressure monitoring is usually performed approximately every half hour. From the mobile network point of view, these parameters have very low throughput requirements. However, the ECG for heartbeat monitoring requires continuous network resources. ECG is a "tracing representing the heart's electrical action derived by amplification of the minutely small electrical impulses normally generated by the heart" [17]. Each beat of the heart is triggered by an electrical impulse normally generated from special cells in the upper right chamber of the heart [18]. An ECG records these electrical signals as they travel through the heart.

The British Heart Foundation (BHF) offers ECG tests where patients are monitored for 24 hours [19]. The electrodes are placed on the chest and the attached wires are connected to a small portable recorder. The test can also be performed while walking on a treadmill or cycling an exercise bike. In special cases, Implantable Loop Recorders (ILRs) are implanted under the patient's skin to monitor the heart activity for up to 14 months. In such cases, it is eminent that the patient would be mobile on several occasions. The ECG data recorder could be provided with mobile connectivity for 
continuous patient diagnosis. The digital data generated by an ECG recorder for transmission over a network is $9.6 \mathrm{kbps}$ according to [20].

\section{Simulation Settings and Results}

The modeling methodology behind our simulation model is to focus primarily on the user plane and evaluate the end-to-end performance. The model is developed using the OPNET simulation environment [21]. In Fig. 1, a number of network elements, e.g. access Gate-Way (aGW), Packet Data Network Gate-Way (PDN GW), eNodeB and the UEs, are depicted. Additionally, some ECG devices are also deployed in the simulation environment. All these network nodes contain the complete protocol stack implementation according to the $3 \mathrm{GPP}$ release 8 specifications. The details of our LTE simulation model are provided in [22], [23]. The OPNET simulations are performed under the parameter settings illustrated in Table 1. Since the ECG data requires uplink radio resources, the Bandwidth and QoS Aware (BQA) LTE uplink scheduler proposed in [24] is used for resource allocation.

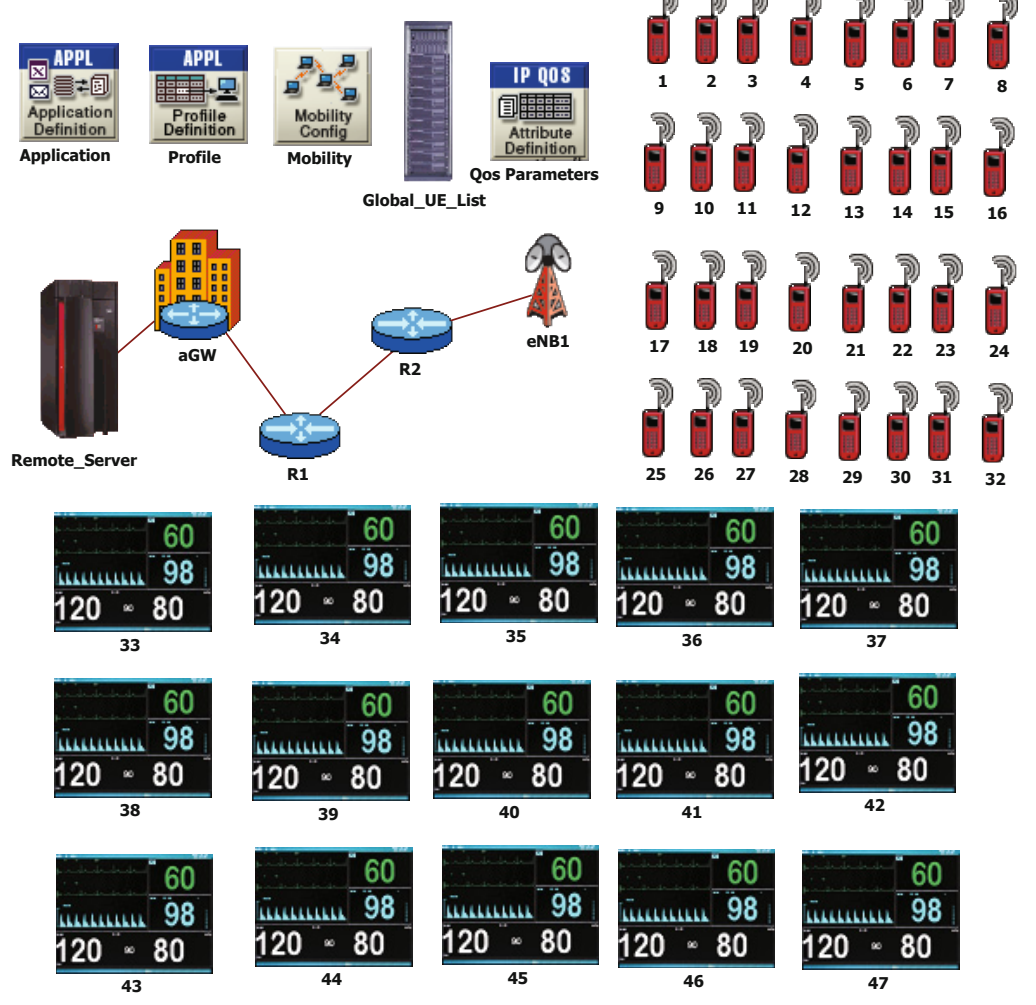

Fig. 1. OPNET LTE simulation model 
Table 1. Simulation Parameters

\begin{tabular}{|c|c|}
\hline Parameter & Setting \\
\hline Cell layout & 1 eNodeB, 3 cells \\
\hline System Bandwidth & $5 \mathrm{MHz}(\sim 25 \mathrm{PRBs})$ \\
\hline Frequency reuse factor & 1 \\
\hline Cell radius & $375 \mathrm{~m}$ \\
\hline Device velocity & $3 \mathrm{kmph}, 120 \mathrm{kmph}$ \\
\hline Max UE power & $23 \mathrm{dBm}$ \\
\hline Path loss & $128.1+37.6 \log _{10}(R), R$ in $\mathrm{km}$ \\
\hline Slow fading & Log-normal shadowing, $8 \mathrm{~dB}$ standard deviation, correlation 1 \\
\hline Fast fading & Jakes-like method [25] \\
\hline Mobility Model & Random Way Point (RWP) \\
\hline UE buffer size & Infinite \\
\hline Power Control & Fractional PC, $\alpha=0.6, P_{0}=-58 \mathrm{dBm}$ \\
\hline Traffic environment & Loaded \\
\hline LTE Uplink Scheduler & BQA [24] \\
\hline \multicolumn{2}{|c|}{ Voice traffic model (Priority 1) } \\
\hline Silence/ talk spurt length & Exponential $(3 \mathrm{sec})$ \\
\hline Encoder scheme & GSM EFR \\
\hline \multicolumn{2}{|c|}{ Video traffic model (Priority 2) } \\
\hline Frame size & 1200 Bytes \\
\hline Frame inter-arrival time & $75 \mathrm{~ms}$ \\
\hline \multicolumn{2}{|c|}{ Mobile point-of-sale traffic model (Priority 3) } \\
\hline Page size & 100 KBytes \\
\hline Page inter-arrival time & $12 \mathrm{sec}$ \\
\hline \multicolumn{2}{|c|}{ FTP traffic model (Priority 4) } \\
\hline File size & 20 MBytes \\
\hline File inter-request time & Uniform distribution, $\min 80 \mathrm{sec}, \max 100 \mathrm{sec}$ \\
\hline \multicolumn{2}{|c|}{ ECG traffic model (Priority 1) } \\
\hline Frame size & 1200 Bytes \\
\hline Frame inter-arrival time & $1 \mathrm{sec}$ \\
\hline
\end{tabular}

The performance of different e-healthcare traffic load scenarios is compared by analyzing the QoS performance of mobile devices with regular LTE uplink traffic. The simulations are carried out by considering four types of LTE uplink traffic, i.e., voice, video, mobile point-of-sale (POS) and file transfer; and one e-healthcare traffic type. The POS traffic has characteristics similar to web browsing, but in uplink direction. So each mobile POS device acts as a web server. The voice and ECG traffic is treated as highest priority traffic by the scheduler. Video traffic has the next highest priority. POS comes after video in terms of priority and web browsing is the best effort traffic with lowest priority. The regular LTE traffic load is kept constant and the ECG data traffic load is varied in the scenarios. In all scenarios, the number of voice, video, mobile POS and file transfer users is 8 each. In the first scenario, there are no 


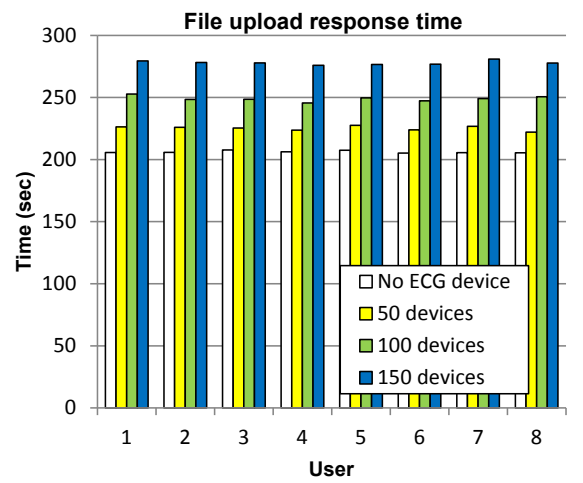

(a)

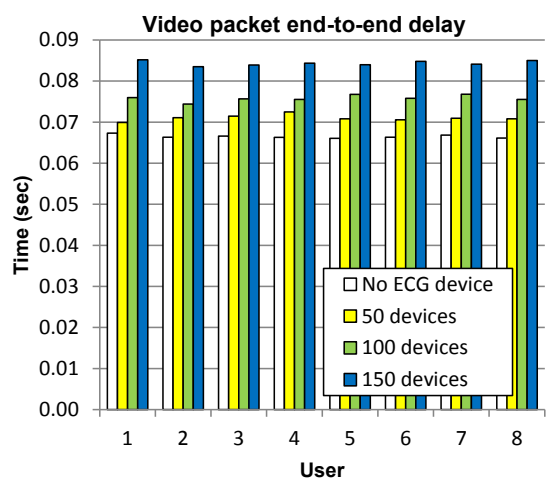

(b)

Fig. 2. (a) Average file upload time (b) Average video user packet end-to-end delay

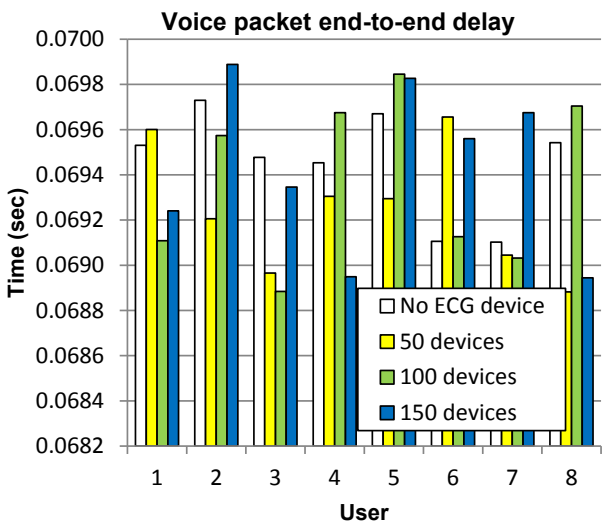

(a)

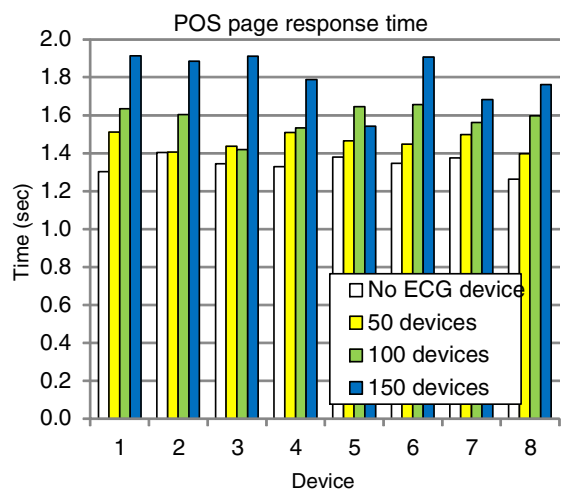

(b)

Fig. 3. (a) Average voice user packet end-to-end delay (b) Average POS page upload time

ECG devices; whereas in the subsequent scenarios, the number of devices is incremented by 50. During the simulations, the velocity of mobile devices and half of the ECG devices deployed in the cell is $120 \mathrm{kmph}$, whereas half of the ECG devices are moving at $3 \mathrm{kmph}$.

The simulation results of LTE regular traffic users are depicted in Fig. 2 (a), Fig. 2 (b) and Fig. 3 (a) for user file upload time, user video packet end-to-end delay and user voice packet end-to-end delay respectively. Fig. 3 (b) illustrates the results for mobile POS devices in terms of device page upload time. There is a clear increase in the traffic delay times of the file upload user, video users and POS devices. The voice user results, however, do not reveal any substantial impact of increasing the ECG traffic load within the LTE cell. The cause that voice traffic is not influenced by the increase in load is that the LTE uplink scheduler [24] gives strict priority to delay sensitive voice traffic. However, the priority of ECG data traffic is higher than other traffic classes. The low priority traffic classes are greatly influenced by the increasing ECG traffic, as evident from the overall average cell QoS results in Fig. 4 (a). Clearly, 

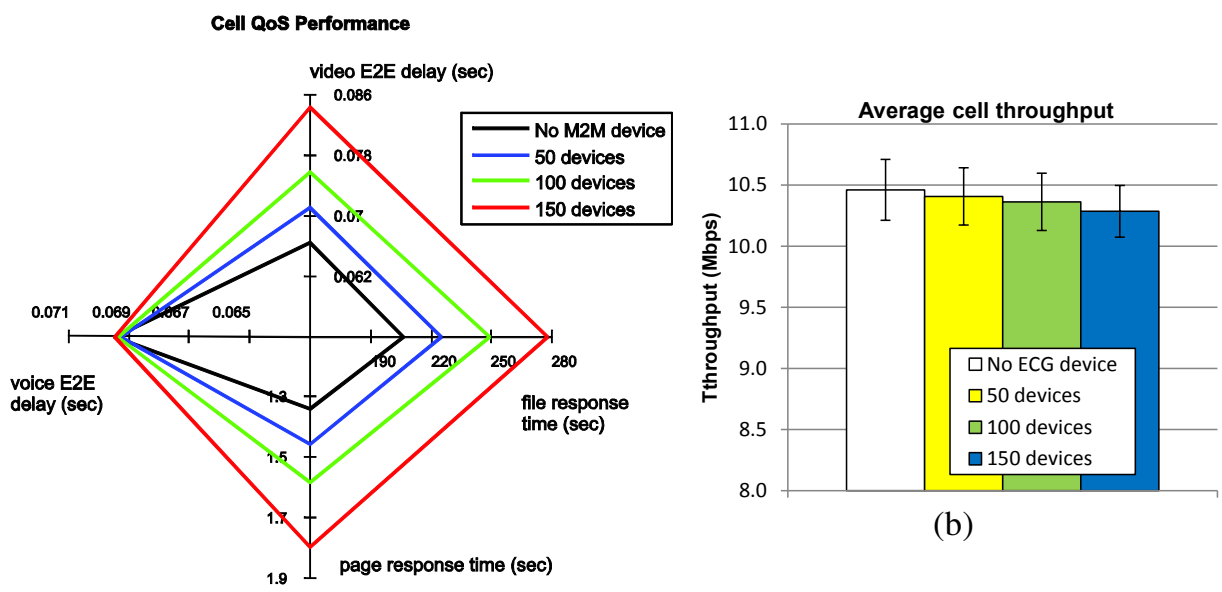

(b)

(a)

Fig. 4. (a) Cell QoS performance comparison with varying load (b) Average cell throughput

the increasing load of ECG traffic drastically degrades the performance of the video, file transfer and POS traffic. Even in such scenarios with low LTE traffic, ECG devices are already causing hindrance in their QoS performance. The results for average cell throughput in Fig. 4 (b) also indicate performance degradation with increasing e-healthcare load.

\section{Conclusion and Outlook}

We conducted a feasibility study in this work to highlight the influence of the new and challenging topic of e-healthcare over the future mobile networks. Though the design of LTE fits the wideband traffic demands, it is not proven how LTE handles the massive deployment of e-healthcare communicating devices. Contrary to the idea that the low M2M traffic might have a negligible impact on future wireless communication systems, we demonstrated in this work that the M2M traffic can have drastic impact on the performance of regular LTE traffic in terms of QoS and throughput.

In future, network operators have to find ways to deal with the issue of increasing M2M and e-healthcare devices; and minimize its influence on the regular LTE traffic. Despite the fact that the performance of voice users remained unaffected by the additional M2M traffic in our simulations, other LTE traffic experienced a considerable increase in delay. We also intend to evaluate the system performance for other kinds of M2M traffic, such as smart metering, intelligent transport, emergency alerting etc. and propose ways to deals with the expected M2M traffic growth in coming years.

Acknowledgement. We are grateful to the International Graduate School for Dynamics in Logistics, University of Bremen, Germany and University of Engineering and Technology, Peshawar, Pakistan for supporting this research work financially. We are also thankful to Dr. Haroon Ahmed Khan, Post Graduate Trainee, Department of General Surgery, Saidu Teaching Hospital, Swat, Pakistan for his assistance in understanding several e-healthcare aspects and ECG functionalities. 


\section{References}

1. Cisco Systems Inc., Cisco Visual Networking Index: Global Mobile Data Traffic Forecast Update, 2011-2016. Digital Publication (February 2012)

2. Coetzee, L., Eksteen, J.: The Internet of Things - promise for the future? An introduction. In: IST-Africa Conference Proceedings, Pretoria, South Africa, May 11-13, pp. 1-9 (2011)

3. Shin, S.H., et al.: Intelligent M2M network using healthcare sensors. In: 14th Asia-Pacific Network Operations and Management Symposium, September 25-27, pp. 1-4 (2012)

4. Chang, Y.-C., Chi, T.-Y., Wang, W.-C., Kuo, S.-Y.: Dynamic software update model for remote entity management of machine-to-machine service capability. IET Communications 7(1), 32-39 (2013)

5. Yunoki, S., Takada, M., Liu, C.: Experimental results of remote energy monitoring system via cellular network in China. In: Proceedings of SICE Annual Conference, Tokyo, Japan, August 20-23, pp. 948-954 (2012)

6. Exalted, http://www.ict-exalted.eu/fileadmin/documents/EXALTED _WP2_D2.1.pdf (Accessed: May 8, 2013)

7. Health Information and Management Systems Society, http://himss.files.cmsplus.com/HIMSSorg/Content/files/ehealth_whitepaper.pdf (Accessed: May 8, 2013)

8. 3GPP Technical Specification $25.913 \mathrm{~V}$ 9.0.0, Requirements for Evolved UTRA (E-UTRA) and Evolved UTRAN (E-UTRAN), (December 2011)

9. Nguyen, T.-D., AI-Saffar, A., Huh, E.-N.: A dynamic ID-based authentication scheme. In: Sixth International Conference on Networked Computing and Advanced Information Management, Suwon, South Korea, August 16-18, pp. 248-253 (2010)

10. LTE for Devices: Requirements, Deployment Phases and Target Scenarios. In: 11th European Wireless Conference 2011 - Sustainable Wireless Technologies, Vienna, Austria, April 27-29, pp. 1-6 (2011)

11. Lu, R., Li, X., Liang, X., Shen, X., Lin, X.: GRS: The green, reliability, and security of emerging machine to machine communications. IEEE Communications Magazine 49(4), 28-35 (2011)

12. Suganthi, J., Umareddy, N.V., Awasthi, N.: Medical alert systems with TeleHealth \& telemedicine monitoring using GSM and GPS technology. In: Third International Conference on Computing Communication \& Networking Technologies, Coimbatore, India, July 26-28, pp. 1-5 (2012)

13. Shin, S.H., et al.: Intelligent M2M network using healthcare sensors. In: 14th Asia-Pacific Network Operations and Management Symposium, Seoul, South Korea, September 25-27, pp. 1-4 (2012)

14. Imadali, S., et al.: eHealth service support in IPv6 vehicular networks. In: IEEE 8th International Conference on Wireless and Mobile Computing, Networking and Communications, Barcelona, Spain, October 8-10, pp. 579-585 (2012)

15. Chen, H., Jia, X.: New requirements and trends of mHealth. In: IEEE 14th International Conference on e-Health Networking, Applications and Services, Beijing, China, October 10-13, pp. 27-31 (2012)

16. Poenaru, E., Poenaru, C.: Networking architectures for healthcare wireless sensor networks comparison of architectures used for remote patient monitoring. In: 11th Roedunet International Conference, Sinaia, Romania, January 17-19, pp. 1-6 (2013)

17. The Free Medical Online Dictionary, http://medical-dictionary. thefreedictionary.com/ECG (Accessed: May 15, 2013) 
18. MayoClinic.com, http://www. mayoclinic.com/health/electrocardiogram/ MY0 0086 (Accessed: May 15, 2013)

19. British Heart Foundation, http://www.bhf.org.uk/heart-health/tests/ ecg. aspx (Accessed: May 15, 2013)

20. Keong, H.C., Yuce, M.R.: Low data rate ultra wideband ECG monitoring system. In: 30th Annual International Conference of the IEEE Engineering in Medicine and Biology Society, Vancouver, Canada, August 20-25, pp. 3413-3416 (2008)

21. OPNET Modeler, http: / /www. opnet. com (Accessed: May 15, 2013)

22. Zaki, Y., Weerawardane, T., Goerg, C., Timm-Giel, A.: Long Term Evolution (LTE) model development within OPNET simulation environment. In: OPNET Workshop, Washington, D.C., USA, August 29-September 1 (2011)

23. Zaki, Y., Zahariev, N., Weerawardane, T., Goerg, C., Timm-Giel, A.: Optimized Service Aware LTE MAC Scheduler: Design, Implementation. In: OPNET Workshop, Washington, D.C., USA, August 29-September 1 (2011)

24. Marwat, S.N.K., Weerawardane, T., Zaki, Y., Goerg, C., Timm-Giel, A.: Performance evaluation of bandwidth and qoS aware LTE uplink scheduler. In: Koucheryavy, Y., Mamatas, L., Matta, I., Tsaoussidis, V. (eds.) WWIC 2012. LNCS, vol. 7277, pp. 298-306. Springer, Heidelberg (2012)

25. Cavers, J.K.: Mobile Channel Characteristics. Kluwer Academic Publishers (2002) 